\section{Generalized Selection of Complementary Matrices in the Inclusion Principle}

\author{
Lubomír Bakule, José Rodellar, and Josep M. Rossell
}

\begin{abstract}
This note presents a strategy for choosing complementary matrices in the framework of the Inclusion Principle with state LQ optimal control of LTI systems. It is based on translating the basic restrictions given by the Inclusion Principle into explicit block structures for these matrices. The degree of freedom given by these structures is illustrated by means of an example of overlapping decentralized control design.
\end{abstract}

Index Terms-Decentralization, inclusion principle, large-scale systems, optimal control, overlapping decomposition.

\section{INTRODUCTION}

The Inclusion Principle was proposed in the early 1980's in the context of analysis and control of complex and large scale systems [4]-[8], [11]. Essentially, the Inclusion Principle establishes a mathematical framework for two dynamic systems with different dimensions, in which solutions of the system with larger-dimension include solutions of the system with smaller dimension. This framework relies on the choice of appropriate linear transformations between the inputs, states and outputs of both systems, which have the freedom of the selection of the so-called complementary matrices. Basically, the idea is to expand a system with overlapped components into a larger-dimensional system that appears decomposed into a number of disjoint subsystems. Then, decentralized controllers are designed for the expanded system and contracted to be implemented in the original system [1], [2], [4], [6], [7], [11].

The conditions given in previous works [4]-[8] on the complementary matrices to ensure the Inclusion Principle have a fundamental, implicit nature, in the sense that they have the form of matrix products from which it is not easy to select specific values for the matrices. In fact, a few simple standard choices have been commonly used in practice, while the exploitation of the degree of freedom offered by the selection of the complementary matrices has been considered as one of interesting research issues, [5]. In this direction, a new characterization of the complementary matrices has been recently presented in [3] and [10], which gives a more explicit way for their selection. It relies on introducing appropriate changes of basis in the expansion-contraction process as already suggested in [8]. This note progresses in the same direction, now with the emphasis on overlapping decentralized state LQ optimal control.

\section{A. Outline of the Paper}

This note contributes with a strategy in choosing the complementary matrices involved in the expansion-contraction process with state LQ

Manuscript received January 2, 1999; revised July 29, 1999 and December 29, 1999. Recommended by Associate Editor, I. Lasiecka. The work of L. Bakule was supported by the Academy of Sciences of the Czech Republic under Grants A2075802 and K1075601. The work of J. Rodellar and J. M. Rossell was supported by the Committee for Science and Technology (CICYT) of Spain under Project TAP99-1079-C03-02.

L. Bakule is with the Institute of Information Theory and Automation, Academy of Sciences of the Czech Republic, 18208 Prague 8, Czech Republic.

J. Rodellar is with the Department of Applied Mathematics III, Universitat Politècnica de Catalunya (UPC), Campus Nord, C-2, 08034-Barcelona, Spain (e-mail: jose.rodellar@upc.es).

J. M. Rossell is with the Department of Applied Mathematics III, Universitat

Politècnica de Catalunya (UPC), 08240-Manresa, Spain.

Publisher Item Identifier S 0018-9286(00)06085-2. optimal control for linear time-invariant systems, including the contractibility conditions.

This note is organized as follows. The problem is formulated in Section II. The main results are presented in Section III, identifying a new block structure of the complementary matrices that generalizes well-known results for expansion-contraction of pairs of systems and optimal control criteria. From this structure, Section IV outlines a selection procedure for the matrices. In Section V, this procedure is used in an overlapping decentralized state LQ optimal control problem, which is illustrated by a numerical example.

\section{PROBLEM Formulation}

Let us start with necessary preliminaries first. Then, the problem is formulated.

\section{A. Preliminaries}

Consider a pair of optimal control problems

$$
\begin{aligned}
\min _{u} J\left(x_{0}, u\right) & =\int_{0}^{\infty}\left(x^{t} Q^{*} x+u^{t} R^{*} u\right) d t, \\
\text { s.t. } \quad \mathbf{S}: \dot{x} & =A x+B u \\
\min _{\tilde{u}} \tilde{J}\left(\tilde{x}_{0}, \tilde{u}\right) & =\int_{0}^{\infty}\left(\tilde{x}^{t} \tilde{Q}^{*} \tilde{x}+\tilde{u}^{t} \tilde{R}^{*} \tilde{u}\right) d t, \\
\text { s.t. } \quad \tilde{\mathbf{S}}: \dot{\tilde{x}} & =\tilde{A} \tilde{x}+\tilde{B} \tilde{u}
\end{aligned}
$$

where $x(t) \in \mathbb{R}^{\mathbf{n}}, u(t) \in \mathbb{R}^{m}$ are the state and input of $\mathbf{S}$ at time $t \in \mathbb{R}^{+}$, and $\tilde{x}(t) \in \mathbb{R}^{\tilde{\mathbf{n}}}, \tilde{u}(t) \in \mathbb{R}^{\tilde{\mathbf{m}}}$ are those ones of $\tilde{\mathbf{S}} . A, B, Q^{*}, R^{*}$ and $\tilde{A}, \tilde{B}, \tilde{Q}^{*}, \tilde{R}^{*}$ are constant matrices of dimensions $n \times n, n \times m$, $n \times n, m \times m$, and $\tilde{n} \times \tilde{n}, \tilde{n} \times \tilde{m}, \tilde{n} \times \tilde{n}, \tilde{m} \times \tilde{m}$, respectively. Matrices $Q^{*} \geq 0, R^{*}>0, \tilde{Q}^{*} \geq 0, \tilde{R}^{*}>0$. Suppose that the dimensions of the state and input vectors $x, u$ of $\mathbf{S}$ are smaller than (or at most equal to) those of $\tilde{x}, \tilde{u}$ of $\tilde{\mathbf{S}}$. Denote $x\left(t ; x_{0}, u\right)$ the state behavior of $\mathbf{S}$ for a fixed input $u(t)$ and an initial state $x(0)=x_{0}$. Similar notation $\tilde{x}\left(t ; \tilde{x}_{0}, \tilde{u}\right)$ is used for the state behavior of system $\tilde{\mathbf{S}}$.

The systems $\mathbf{S}$ and $\tilde{\mathbf{S}}$ are related by the transformations $\tilde{x}=V x$, $x=U \tilde{x}, \tilde{u}=R u, u=Q \tilde{u}$, where $V$ and $R$ are constant matrices of appropriate dimensions and full rank. $U$ and $Q$ are constant matrices of appropriate dimensions and full rank, satisfying $U V=I_{n}, Q R=I_{m}$ where $I_{n}, I_{m}$ are identity matrices of indicated dimensions [11].

Definition 1 (Inclusion Principle): We say that the system $\tilde{\mathbf{S}}$ includes the system $\mathbf{S}$, that is $\tilde{\mathbf{S}} \supset \mathbf{S}$, if there exists a triplet $(U, V, R)$ such that, for any initial state $x_{0}$ and any fixed input $u(t)$ of $\mathbf{S}$, the choice $\tilde{x}_{0}=V x_{0}$ and $\tilde{u}(t)=R u(t)$ for all $t \geq 0$ of the initial state $\tilde{x}_{0}$ and input $\tilde{u}(t)$ of the system $\tilde{\mathbf{S}}$ implies $x\left(t ; x_{0}, u\right)=U \tilde{x}\left(t ; \tilde{x}_{0}, \tilde{u}\right)$, for all $t \geq 0$.

If $\tilde{\mathbf{S}} \supset \mathbf{S}$, then $\tilde{\mathbf{S}}$ it is said to be an expansion of $\mathbf{S}$ and $\mathbf{S}$ is called a contraction of $\tilde{\mathbf{S}}$.

Definition 2: We say that the pair $(\tilde{\mathbf{S}}, \tilde{J})$ includes the pair $(\mathbf{S}, J)$, that is $(\tilde{\mathbf{S}}, \tilde{J}) \supset(\mathbf{S}, J)$, if $\tilde{\mathbf{S}} \supset \mathbf{S}$ and $J\left(x_{0}, u\right)=\tilde{J}\left(\tilde{x}_{0}, \tilde{u}\right)$.

The matrices of $(\tilde{\mathbf{S}}, \tilde{J})$ and $(\mathbf{S}, J)$ can be related as $\tilde{A}=V A U+M$, $\tilde{B}=V B Q+N, \tilde{Q}^{*}=U^{t} Q^{*} U+M_{Q^{*}}$ and $\tilde{R}^{*}=Q^{t} R^{*} Q+N_{R^{*}}$, where $M, N, M_{Q^{*}}$, and $N_{R^{*}}$ are constant complementary matrices of appropriate dimensions.

Theorem 1: The system $\tilde{\mathbf{S}}$ is an expansion of the system $\mathbf{S}$ if and only if $U M^{i} V=0$ and $U M^{i-1} N R=0$ for all $i=1,2, \cdots, \tilde{n}$.

Theorem 2: $\tilde{\mathbf{S}} \supset \mathbf{S}$ if and only if there exists $\overline{\mathbf{S}}$ such that $\tilde{\mathbf{S}} \supset \overline{\mathbf{S}} \supset$ $\mathbf{S}$, where $\overline{\mathbf{S}}$ is a restriction (aggregation) of $\tilde{\mathbf{S}}$ and $\mathbf{S}$ is an aggregation (restriction) of $\overline{\mathbf{S}}$.

For $(\tilde{\mathbf{S}}, \tilde{J})$ to be an expansion of $(\mathbf{S}, J)$, a proper choice of $M, N$, $M_{Q^{*}}$, and $N_{R^{*}}$ is required. It is provided by the following theorem [7]. 
Theorem 3: The pair $(\tilde{\mathbf{S}}, \tilde{J}) \supset(\mathbf{S}, J)$ if either

1) $M V=0, N R=0, V^{t} M_{Q^{*}} V=0, R^{t} N_{R^{*}} R=0$ or

2) $U M^{i} V=0, U M^{i-1} N R=0, M_{Q^{*}} M^{i-1} V=0$, $M_{Q^{*}} M^{i-1} N R=0, R^{t} N_{R^{*}} R=0$ for all $i=1,2, \cdots, \tilde{n}$.

Definition 3: A control law $\tilde{u}=-\tilde{K} \tilde{x}$ for $\tilde{\mathbf{S}}$ is contractible to the control law $u=-K x$ for implementation in $\mathbf{S}$ if the choice $\tilde{x}_{0}=V x_{0}$ and $\tilde{u}=R u$ implies $K x\left(t ; x_{0}, u\right)=Q \tilde{K} \tilde{x}\left(t ; \tilde{x}_{0}, \tilde{u}\right)$, for all $t \geq 0$, for any initial state $x_{0}$ and any fixed input $u(t)$ of $\mathbf{S}$.

Suppose $\tilde{K}=R K U+F$, where $F$ denotes a complementary matrix. The conditions to satisfy Definition 3 are given by the following theorem [7].

Theorem 4: A control law $\tilde{u}=-\tilde{K} \tilde{x}$ for $\tilde{\mathbf{S}}$ is contractible to the control law $u=-K x$ for $\mathbf{S}$ if and only if $Q F M^{i-1} V=0$ and $Q F M^{i-1} N R=0$, for all $i=1,2, \cdots, \tilde{n}$.

An equivalent form of Theorem 4 , which gives an explicit expression of matrix $K$ from matrix $\tilde{K}$, is the following.

Theorem 5: A control law $\tilde{u}=-\tilde{K} \tilde{x}$ for $\tilde{\mathbf{S}}$ is contractible to the control law $u=-K x$ for $\mathbf{S}$ if and only if $Q \tilde{K} V=K, Q \tilde{K} M^{i-1} V=$ 0 for all $i=2, \cdots, \tilde{n}$, and $Q \tilde{K} M^{i-1} N R=0$ for all $i=1,2, \cdots, \tilde{n}$.

\section{B. The Problem}

The motivation of this note is to extend systematically the class of complementary matrices to obtain a more flexible selection strategy in the context summarized above. The specific goals of this note are the following:

- To derive a systematic procedure of selection of the complementary matrices in any expansion-contraction process for the pairs $(\tilde{\mathbf{S}}, \tilde{J}) \supset(\mathbf{S}, J)$, including the contractibility conditions, based on a new block structure characterization of such matrices.

- To use this procedure in expansion-contraction process for the pairs $(\mathbf{S}, J),(\tilde{\mathbf{S}}, \tilde{J})$ involving overlapping decentralized state LQ optimal control design.

- To verify the derived results on a numerical example.

\section{EXPANSION-CONTRACTION PROCESS}

\section{A. Change of Basis}

The expansion and contraction between systems $\mathbf{S}$ and $\tilde{\mathbf{S}}$ can be illustrated in the form

$$
\begin{gathered}
\mathbf{S} \rightarrow \tilde{\mathbf{S}} \rightarrow \mathbf{S} \\
\mathbb{R}^{\mathbf{n}} \stackrel{V}{\rightarrow} \mathbb{R}^{\tilde{\mathbf{n}}} \stackrel{U}{\rightarrow} \mathbb{R}^{\mathbf{n}} \\
\mathbb{R}^{\mathbf{m}} \stackrel{R}{\rightarrow} \mathbb{R}^{\tilde{\mathbf{m}}} \stackrel{Q}{\rightarrow} \mathbb{R}^{\mathbf{m}} .
\end{gathered}
$$

Since the Inclusion Principle does not depend on the specific basis used in the state, input and output spaces for both systems $\mathbf{S}$ and $\tilde{\mathbf{S}}$, we may introduce convenient changes of basis in $\tilde{\mathbf{S}}$ [3], [10]. Thus, the above scheme can be modified in the form

$$
\begin{gathered}
\mathbf{S} \rightarrow \tilde{\mathbf{S}} \rightarrow \tilde{\overline{\mathbf{S}}} \rightarrow \tilde{\mathbf{S}} \rightarrow \mathbf{S}, \\
\mathbb{R}^{\mathbf{n}} \stackrel{V}{\rightarrow} \mathbb{R}^{\tilde{\mathbf{n}}} \stackrel{T_{A}^{-1}}{\rightarrow} \overline{\mathbb{R}}^{\tilde{\mathbf{n}}} \stackrel{T_{A}}{\rightarrow} \mathbb{R}^{\tilde{\mathbf{n}}} \stackrel{U}{\rightarrow} \mathbb{R}^{\mathbf{n}} \\
\mathbb{R}^{\mathbf{m}} \stackrel{R}{\rightarrow} \mathbb{R}^{\tilde{\mathbf{m}}} \stackrel{T_{B}^{-1}}{\rightarrow} \overline{\mathbb{R}}^{\tilde{\mathbf{m}}} \stackrel{T_{B}}{\rightarrow} \mathbb{R}^{\tilde{\mathbf{m}}} \stackrel{Q}{\rightarrow} \mathbb{R}^{\mathbf{m}}
\end{gathered}
$$

where $\tilde{\overline{\mathbf{S}}}$ denotes the expanded system with the new basis. The idea of using changes of basis in the expansion-contraction process was already introduced by Ikeda et al. [8] to represent $\tilde{\mathbf{S}}$ in a canonical form (Theorem 2). Given $V$ and $R$, we define $U=\left(V^{t} V\right)^{-1} V^{t}$, $Q=\left(R^{t} R\right)^{-1} R^{t}$ as their pseudoinverses, respectively. Let us consider

$$
T_{A}=\left(\begin{array}{ll}
V & W_{A}
\end{array}\right), \quad T_{B}=\left(\begin{array}{ll}
R & W_{B}
\end{array}\right)
$$

where $W_{A}, W_{B}$ are chosen such that $\operatorname{Im} W_{A}=\operatorname{Ker} U, \operatorname{Im} W_{B}=$ $\operatorname{Ker} Q$. Using these transformations it is easy to verify the conditions

$$
\overline{U V}=I_{n}, \quad \overline{V U}=\left(\begin{array}{cc}
I_{n} & 0 \\
0 & 0
\end{array}\right)
$$

and

$$
\overline{Q R}=I_{m}, \quad \overline{R Q}=\left(\begin{array}{cc}
I_{m} & 0 \\
0 & 0
\end{array}\right)
$$

where

$$
\begin{aligned}
& \bar{V}=T_{A}^{-1} V=\left(\begin{array}{c}
I_{n} \\
0
\end{array}\right) \\
& \bar{U}=U T_{A}=\left(\begin{array}{ll}
I_{n} & 0
\end{array}\right)
\end{aligned}
$$

and

$$
\bar{R}=T_{B}^{-1} R=\left(\begin{array}{c}
I_{m} \\
0
\end{array}\right), \quad \bar{Q}=Q T_{B}=\left(\begin{array}{ll}
I_{m} & 0
\end{array}\right) .
$$

In fact, obtaining these conditions is the motivating factor for defining $T_{A}$ and $T_{B}$ in (5). As we are going to develop in the remainder of this section, these conditions will be crucial to obtain explicit block structures (with zero blocks) of the complementary matrices and further to give a general strategy for their selection in Section IV.

\section{B. Expansion-Contraction in the New Basis}

The expansion-contraction process will be developed for the system $\mathbf{S}$ having the following structure:

$$
\begin{aligned}
\mathbf{S :}\left(\begin{array}{l}
\dot{x}_{1} \\
\dot{x}_{2} \\
\dot{x}_{3}
\end{array}\right)= & \left(\begin{array}{c|c|c}
A_{11} & A_{12} & A_{13} \\
& ---- & ---- \\
A_{21} & A_{22} & A_{23} \\
---- & ---- & \\
A_{31} & A_{32} & A_{33}
\end{array}\right)\left(\begin{array}{l}
x_{1} \\
x_{2} \\
x_{3}
\end{array}\right) \\
& +\left(\begin{array}{c|c|c}
B_{11} & B_{12} & B_{13} \\
B_{21} & ---- & ---- \\
---- & ---- & B_{22} \\
B_{31} & B_{32} & B_{33}
\end{array}\right)\left(\begin{array}{l}
u_{1} \\
u_{2} \\
u_{3}
\end{array}\right)
\end{aligned}
$$

where $A_{i i}, B_{i i}, i=1,2,3$, are $n_{i} \times n_{i}$ and $n_{i} \times m_{i}$ dimensional matrices, respectively. This system is composed of two subsystems with one overlapped part. This simple structure will help in smoothing the notation. The results obtained can be easily generalized for any number of interconnected overlapping subsystems. This structure has been extensively adopted as prototype in the literature within the Inclusion Principle.

Consider the optimal control problem in the system $\overline{\mathbf{S}}$

$$
\begin{aligned}
& \min _{\tilde{\tilde{u}}} \tilde{\bar{J}}\left(\tilde{\bar{x}}_{0}, \tilde{\bar{u}}\right)=\int_{0}^{\infty}\left(\tilde{\bar{x}}^{t} t \overline{\bar{Q}}^{*} \frac{\tilde{\bar{x}}}{\tilde{\bar{u}}^{t}} \tilde{\bar{R}}^{*} \tilde{\bar{u}}\right) d t \\
& \text { s.t. } \quad \tilde{\overline{\mathbf{S}}}: \dot{\bar{x}}=\tilde{\bar{A}} \tilde{\bar{x}}+\tilde{\bar{B}} \tilde{\bar{u}}
\end{aligned}
$$

where $\tilde{\bar{x}}$ and $\tilde{\bar{u}}$ are defined as $\tilde{\bar{x}}=T_{A}^{-1} V x=\bar{V} x, \tilde{\bar{u}}=T_{B}^{-1} R u=\bar{R} u$. $\tilde{\bar{A}}, \tilde{\bar{B}}, \tilde{\bar{Q}}^{*}$, and $\tilde{\bar{R}}^{*}$ are constant matrices of appropriate dimensions verifying $\overline{\bar{A}}=\bar{V} A \bar{U}+\bar{M}, \bar{B}=\bar{V} B \bar{Q}+\bar{N}, \bar{Q}^{*}=\bar{U}^{t} Q^{*} \bar{U}+\bar{M}_{Q^{*}}$, $\tilde{\bar{R}}^{*}=\bar{Q}^{t} R^{*} \bar{Q}+\bar{N}_{R^{*}}$, where the new complementary matrices are $\bar{M}=T_{A}^{-1} M T_{A}, \bar{N}=T_{A}^{-1} N T_{B}, \bar{M}_{Q^{*}}=T_{A}^{t} M_{Q^{*}} T_{A}$, $\bar{N}_{R^{*}}=T_{B}^{t} N_{R^{*}} T_{B}$. Using these matrices, the conditions given by the Inclusion Principle (Theorem 1) become $\overline{U M} \bar{V}=0$ and $\overline{U M}^{i-1} \bar{N} \bar{R}=0$ for all $i=1,2, \cdots, \tilde{n}$. First, we analyze the form of matrices $\bar{M}, \bar{N}, \bar{M}_{Q^{*}}$, and $\bar{N}_{R^{*}}$. Consider the complementary matrices $M=\left(M_{i j}\right), N=\left(N_{i j}\right), M_{Q^{*}}=\left(M_{Q_{i j}^{*}}\right), N_{R^{*}}=$ 
$\left(N_{R_{i j}^{*}}\right), i, j=1, \cdots, 4$, where each submatrix has appropriate dimensions and $M_{Q_{i j}^{*}}=M_{Q_{j i}^{*}}^{t}, N_{R_{i j}^{*}}=N_{R_{j i}^{*}}^{t}$. Consider the matrices

$$
\begin{aligned}
\bar{M} & =\left(\begin{array}{ll}
\bar{M}_{11} & \bar{M}_{12} \\
\bar{M}_{21} & \bar{M}_{22}
\end{array}\right) \\
\bar{N} & =\left(\begin{array}{ll}
\bar{N}_{11} & \bar{N}_{12} \\
\bar{N}_{21} & \bar{N}_{22}
\end{array}\right) \\
\bar{M}_{Q^{*}} & =\left(\begin{array}{ll}
\bar{M}_{Q_{11}^{*}} & \bar{M}_{Q_{12}^{*}} \\
\bar{M}_{Q_{12}^{*}}^{t} & \bar{M}_{Q_{22}^{*}}
\end{array}\right) \\
\bar{N}_{R^{*}} & =\left(\begin{array}{ll}
\bar{N}_{R_{11}^{*}} & \bar{N}_{R_{12}^{*}} \\
\bar{N}_{R_{12}^{*}}^{t} & \bar{N}_{R_{22}^{*}}
\end{array}\right)
\end{aligned}
$$

where $\bar{M}_{11}, \bar{M}_{22}$ are $n \times n,(\tilde{n}-n) \times(\tilde{n}-n)$-dimensional matrices, respectively. $\bar{N}_{11}, \bar{N}_{22}$ are $n \times m,(\tilde{n}-n) \times(\tilde{m}-m)$-dimensional matrices, respectively. $\bar{M}_{Q_{11}^{*}}, \bar{M}_{Q_{22}^{*}}$ are $n \times n,(\tilde{n}-n) \times(\tilde{n}-n)$-dimensional matrices, respectively, and $\bar{N}_{R_{11}^{*}}, \bar{N}_{R_{22}^{*}}$ are $m \times m,(\tilde{m}-$ $m) \times(\tilde{m}-m)$-dimensional matrices, respectively. We need to know the form of the submatrices $\bar{M}_{i j}, \bar{N}_{i j}, \bar{M}_{Q_{i, j}^{*}}$, and $\bar{N}_{R_{i, j}^{*}}$ for all $i, j=1,2$. This is given by the following propositions.

Proposition 1: Consider the system given by (1) and the corresponding expanded system (7) verifying the Inclusion Principle. Then,

$$
\bar{M}=\left(\begin{array}{cc}
0 & \bar{M}_{12} \\
\bar{M}_{21} & \bar{M}_{22}
\end{array}\right)
$$

where (0) denotes a matrix of order $n$ and the other blocks satisfy $\bar{M}_{12} \bar{M}_{22}^{i-2} \bar{M}_{21}=0$ for all $i=2, \cdots, \tilde{n}$.

Proof: Let $\bar{M}$ be

$$
\bar{M}=\left(\begin{array}{ll}
\bar{M}_{11} & \bar{M}_{12} \\
\bar{M}_{21} & \bar{M}_{22}
\end{array}\right) .
$$

Imposing $\overline{U M}^{i} \bar{V}=0$ for $i=1$, we get $\overline{U M V}=0$, so that $\bar{M}_{11}=$ 0 . The powers of $\bar{M}$ for $\mu=2, \cdots, \tilde{n}$ result in (8) shown at the bottom of the page. Then, $\overline{U M}^{k} \bar{V}=0$, for $i=k \geq 2$, implies that $\bar{M}_{12} \bar{M}_{22}^{k-2} \bar{M}_{21}=0$. Repeating this process $\overline{U M^{\tilde{n}}} \bar{V}=0$, for $i=\tilde{n}$, leads to $\bar{M}_{12} \bar{M}_{22}^{\tilde{n}-2} \bar{M}_{21}=0$.

Proposition 2: Consider the system given by (1) and the corresponding expanded system (7) verifying the Inclusion Principle. Then,

$$
\bar{N}=\left(\begin{array}{cc}
0 & \bar{N}_{12} \\
\bar{N}_{21} & \bar{N}_{22}
\end{array}\right)
$$

where $(0)$ denotes an $n \times m$-dimensional matrix in $\bar{N}$ and the other blocks satisfy $\bar{M}_{12} \bar{M}_{22}^{i-2} \bar{N}_{21}=0$ for all $i=2, \cdots, \tilde{n}$.

Proof: This proof is similar to Proposition 1 by using the condition $\overline{U M}^{i-1} \overline{N R}=0$.

Remark: The conditions imposed by the Inclusion Principle (Theorem 1) have been reduced to the more explicit conditions on submatrices in the form $\bar{M}_{12} \bar{M}_{22}^{i-2} \bar{M}_{21}=0, \bar{M}_{12} \bar{M}_{22}^{i-2} \bar{N}_{21}=0$, for all $i=2, \cdots, \tilde{n}$, with

$$
\bar{M}=\left(\begin{array}{cc}
0 & \bar{M}_{12} \\
\bar{M}_{21} & \bar{M}_{22}
\end{array}\right) \quad \text { and } \quad \bar{N}=\left(\begin{array}{cc}
0 & \bar{N}_{12} \\
\bar{N}_{21} & \bar{N}_{22}
\end{array}\right) .
$$

Theorem 6: Consider the problems (1) and (7). Then, the pair $(\overline{\mathbf{S}}, \bar{J}) \supset(\mathbf{S}, J)$ if either

1)

$$
\begin{aligned}
\bar{M} & =\left(\begin{array}{ll}
0 & \bar{M}_{12} \\
0 & \bar{M}_{22}
\end{array}\right) \\
\bar{N} & =\left(\begin{array}{ll}
0 & \bar{N}_{12} \\
0 & \bar{N}_{22}
\end{array}\right) \\
\bar{M}_{Q^{*}} & =\left(\begin{array}{cc}
0 & \bar{M}_{Q_{12}^{*}} \\
\bar{M}_{Q_{12}^{*}}^{t} & \bar{M}_{Q_{22}^{*}}
\end{array}\right) \\
\bar{N}_{R^{*}} & =\left(\begin{array}{cc}
0 & \bar{N}_{R_{12}^{*}} \\
\bar{N}_{R_{12}^{*}}^{t} & \bar{N}_{R_{22}^{*}}
\end{array}\right)
\end{aligned}
$$

or

2)

$$
\bar{M}=\left(\begin{array}{cc}
0 & \bar{M}_{12} \\
\bar{M}_{21} & \bar{M}_{22}
\end{array}\right) \text { and } \bar{N}=\left(\begin{array}{cc}
0 & \bar{N}_{12} \\
\bar{N}_{21} & \bar{N}_{22}
\end{array}\right)
$$

such that $\bar{M}_{12} \bar{M}_{22}^{i-2} \bar{M}_{21}=0$ and $\bar{M}_{12} \bar{M}_{22}^{i-2} \bar{N}_{21}=0$ for all $i=2, \cdots, \tilde{n}$;

$$
\bar{M}_{Q^{*}}=\left(\begin{array}{cc}
0 & 0 \\
0 & \bar{M}_{Q_{22}^{*}}
\end{array}\right)
$$

such that $\bar{M}_{Q_{22}^{*}} \bar{M}_{22}^{i-2} \bar{M}_{21}=0$ for all $i=2, \cdots, \tilde{n}$; $\bar{M}_{Q_{22}^{*}} \bar{M}_{22}^{i-2} \bar{N}_{21}=0$ for all $i=2, \cdots, \tilde{n}+1$; and

$$
\bar{N}_{R^{*}}=\left(\begin{array}{cc}
0 & \bar{N}_{R_{12}^{*}} \\
\bar{N}_{R_{12}^{*}}^{t} & \bar{N}_{R_{22}^{*}}
\end{array}\right) .
$$

Proof: Consider the relations given by Theorem 3. The first block of conditions in Theorem 6 is a consequence of $\overline{M V}=0, \overline{N R}=0, \bar{V}^{t} \bar{M}_{Q *} \bar{V}=0, \bar{R}^{t} \bar{N}_{R}^{*} \bar{R}=0$. From relations $\overline{U M}^{i} \bar{V}=0, \overline{U M}^{i-1} \overline{N R}=0, \bar{M}_{Q^{*}} \bar{M}^{i-1} \bar{V}=$ $0, \bar{M}_{Q^{*}} \bar{M}^{i-1} \overline{N R}=0, \bar{R}^{t} \bar{N}_{R}^{*} \bar{R}=0$, together with Propositions 1 and 2 , we get the second block of conditions.

Let us consider the optimal control laws for $(\tilde{\overline{\mathbf{S}}}, \tilde{\bar{J}})$ and $(\mathbf{S}, J)$. Let us denote the corresponding values of the performance criteria as $\tilde{\bar{J}}^{\circ}$ and $J^{\circ}$, respectively. Then the following theorem can be presented.

Theorem 7: The pair $\left(\tilde{\overline{\mathbf{S}}}, \tilde{\bar{J}}^{\circ}\right) \supset\left(\mathbf{S}, J^{\circ}\right)$ if $\overline{M V}=0, R^{*}=$ $\left(\bar{Q}\left(\tilde{\bar{R}}^{*}\right)^{-1} \bar{Q}^{t}\right)^{-1}, \bar{N}=0$ and $\bar{V}^{t} \bar{M}_{Q *} \bar{V}=0$.

Proof: Denote the solutions of the Riccati equations for problems (1) and (7) as $P$ and $\tilde{\bar{P}}$, respectively. Consider the optimal costs for given $x_{0}, J^{\circ}\left(x_{0}\right)=x_{0}^{t} P x_{0}$ and $\tilde{\bar{J}}^{\circ}\left(\tilde{\bar{x}}_{0}\right)=\tilde{\bar{x}}_{0}^{t} \tilde{\bar{P}}_{\overline{x_{0}}}$. The relation

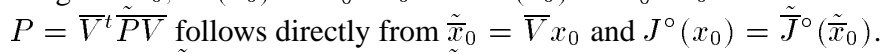
Substituting $\tilde{\bar{A}}=\bar{V} A \bar{U}+\bar{M}$ and $\tilde{\bar{B}}=\bar{V} B \bar{Q}+\bar{N}$ into the Riccati equation of problem (7) and $P=\bar{V}^{t} \overline{\bar{P} V}$ into the Riccati equation of problem (1) and comparing both equations the proof is completed.

\section{Contractibility}

Now, we determine the conditions, in terms of the complementary matrices, when a control law designed in the expanded system $\tilde{\overline{\mathbf{S}}}$ can

$$
\bar{M}^{\mu}=\left(\begin{array}{cc}
\bar{M}_{12} \bar{M}_{22}^{\mu-2} \bar{M}_{21} & \bar{M}_{12} \bar{M}_{22}^{\mu-1} \\
\bar{M}_{22}^{\mu-1} \bar{M}_{21} & \bar{M}_{22}^{\mu}+\sum_{j=0}^{j=\mu-2} \bar{M}_{22}^{j} \bar{M}_{21} \bar{M}_{12} \bar{M}_{22}^{\mu-2-j}
\end{array}\right)
$$


be contracted to be implemented in the initial system $\mathbf{S}$. Let $K$ be the gain matrix for the prototype system (6) and $F$ the corresponding complementary matrix, which has the form $F=\left(F_{i j}\right), i, j=1, \cdots, 4$. Define

$$
\bar{F}=\left(\begin{array}{ll}
\bar{F}_{11} & \bar{F}_{12} \\
\bar{F}_{21} & \bar{F}_{22}
\end{array}\right)
$$

where $\bar{F}_{11}, \bar{F}_{22}$ are $m \times n,(\tilde{m}-m) \times(\tilde{n}-n)$-dimensional matrices, respectively. The gain matrix $\tilde{\bar{K}}$ for the system $\tilde{\overline{\mathbf{S}}}$ has the form $\tilde{\bar{K}}=\bar{R} K \bar{U}+\bar{F}$, where $\tilde{\bar{K}}=T_{B}^{-1} \tilde{K} T_{A}$ and $\bar{F}=T_{B}^{-1} F T_{A}$. By Definition $3, \tilde{\bar{u}}=-\tilde{\bar{K}} \tilde{\bar{x}}$ is contractible to the control law $u=-K x$ if $K x\left(t ; x_{0}, u\right)=\overline{Q K} \tilde{\bar{x}}\left(t ; \bar{V} x_{0}, \bar{R} u\right)$.

Theorem 8: A control law $\tilde{\bar{u}}=-\tilde{\bar{K}} \tilde{\bar{x}}$ designed in the expanded system $\tilde{\mathbf{S}}$ is contractible to the control law $u=-K x$ of the system $\mathbf{S}$ if and only if

$$
\bar{F}=\left(\begin{array}{cc}
0 & \bar{F}_{12} \\
\bar{F}_{21} & \bar{F}_{22}
\end{array}\right)
$$

and $\bar{F}_{12} \bar{M}_{22}^{i-2} \bar{M}_{21}=0, \bar{F}_{12} \bar{M}_{22}^{i-2} \bar{N}_{21}=0$ for all $i=2, \cdots, \tilde{n}+1$.

Proof: It follows from the conditions given by Propositions 1, 2, and Theorem 4.

\section{PRocedure For SELECTION OF COMPLEMENTARY MATRICES}

The expansion-contraction process between problems (1) and (2) involves the complementary matrices $M, N, M_{Q^{*}}, N_{R^{*}}$, and $F$, which must verify the fundamental restrictions given by the well-known Theorems 3 and 4. In this note, by introducing convenient changes of basis in $\tilde{\mathbf{S}}$, a transformed problem (7) is considered, whose relation with the initial problem (1) is through the complementary matrices $\bar{M}, \bar{N}, \bar{M}_{Q^{*}}, \bar{N}_{R^{*}}$, and $\bar{F}$. Propositions 1 and 2 give a characterization of $\bar{M}$ and $\bar{N}$ for $\tilde{\mathbf{S}}$ be an expansion of $\mathbf{S}$. Theorems 6 and 7 characterize $\bar{M}_{Q^{*}}$ and $\bar{N}_{R^{*}}$ involved in the equivalence of the optimal control problems (1) and (7). Theorem 8 gives a characterization of $\bar{F}$ to ensure the contractibility of any controller designed for system $\tilde{\overline{\mathbf{S}}}$. These characterizations give a general block structure of all the complementary matrices independently of the choice of the transformations $V$ and $R$ between the original system $\mathbf{S}$ and its expanded version $\tilde{\mathbf{S}}$. Although the formulation has been developed for the prototype system with the structure (6), the above characterization can be readily generalized for any other system structure.

To make a practical use of these characterizations, we start by defining the specific transformations $V$ and $R$ to expand a given problem (1). The choice of these expansion matrices is limited by the information structure constraints requiring the preservation of the integrity of the local feedback and subsystems in overlapping decentralized control. Once $V$ and $R$ are chosen, the corresponding changes of basis $T_{A}$ and $T_{B}$ are given by (5). It is straightforward to obtain the structure of the complementary matrices $M, N, M_{Q^{*}}, N_{R^{*}}$, and $F$ from the expressions $\bar{M}=T_{A}^{-1} M T_{A}, \bar{N}=T_{A}^{-1} N T_{B}, \bar{M}_{Q^{*}}=$ $T_{A}^{t} M_{Q^{*}} T_{A}, \bar{N}_{R^{*}}=T_{B}^{t} N_{R^{*}} T_{B}$, and $\bar{F}=T_{B}^{-1} F T_{A}$. Finally, from the derived structure, the designer can select specific values of the elements of complementary matrices according to given specifications.

\section{A. Particular Selection}

Let us illustrate this procedure for the following expansion transformation matrices:

$$
V=\left(\begin{array}{ccc}
I_{n_{1}} & 0 & 0 \\
0 & I_{n_{2}} & 0 \\
0 & I_{n_{2}} & 0 \\
0 & 0 & I_{n_{3}}
\end{array}\right), \quad R=\left(\begin{array}{ccc}
I_{m_{1}} & 0 & 0 \\
0 & I_{m_{2}} & 0 \\
0 & I_{m_{2}} & 0 \\
0 & 0 & I_{m_{3}}
\end{array}\right)
$$

These transformations are chosen to lead, in a simple natural way, to an expanded system where state vector $x_{2}$ and control vector $u_{2}$ appear repeated in $\tilde{x}^{t}=\left(x_{1}^{t}, x_{2}^{t}, x_{2}^{t}, x_{3}^{t}\right)$ and $\tilde{u}^{t}=\left(u_{1}^{t}, u_{2}^{t}, u_{2}^{t}, u_{3}^{t}\right)$, respectively. According to (5), the changes of basis to define the system $\overline{\mathbf{S}}$ for matrices (9) are given by

$$
\begin{aligned}
T_{A} & =\left(\begin{array}{cccc}
I_{n_{1}} & 0 & 0 & 0 \\
0 & I_{n_{2}} & 0 & I_{n_{2}} \\
0 & I_{n_{2}} & 0 & -I_{n_{2}} \\
0 & 0 & I_{n_{3}} & 0
\end{array}\right) \\
T_{A}^{-1} & =\left(\begin{array}{cccc}
I_{n_{1}} & 0 & 0 & 0 \\
0 & \frac{1}{2} I_{n_{2}} & \frac{1}{2} I_{n_{2}} & 0 \\
0 & 0 & 0 & I_{n_{3}} \\
0 & \frac{1}{2} I_{n_{2}} & -\frac{1}{2} I_{n_{2}} & 0
\end{array}\right) .
\end{aligned}
$$

Analogously, by $T_{B}, T_{B}^{-1}$. Then, by Propositions 1 and 2, using $\bar{M}=$ $T_{A}^{-1} M T_{A}$ and $\bar{N}=T_{A}^{-1} N T_{B}$, it is easy to obtain the following structure for complementary matrix $M$ :

$$
M=\left(\begin{array}{cccc}
0 & M_{12} & -M_{12} & 0 \\
M_{21} & M_{22} & M_{23} & M_{24} \\
-M_{21} & -\left(M_{22}+M_{23}+M_{33}\right) & M_{33} & -M_{24} \\
0 & M_{42} & -M_{42} & 0
\end{array}\right)
$$

and a similar one for $N$, which, for all $i=2, \cdots, \tilde{n}$, must verify

$$
\begin{aligned}
& \left(\begin{array}{c}
M_{12} \\
M_{23}+M_{33} \\
M_{42}
\end{array}\right) \\
& \times\left(M_{22}+M_{33}\right)^{i-2}\left(\begin{array}{lll}
M_{21} & M_{22}+M_{23} & \left.M_{24}\right)=0 \\
M_{12} \\
M_{23}+M_{33} \\
M_{42}
\end{array}\right) \\
& \times\left(M_{22}+M_{33}\right)^{i-2}\left(\begin{array}{lll}
N_{21} & N_{22}+N_{23} & \left.N_{24}\right)=0 .
\end{array}\right.
\end{aligned}
$$

The corresponding expanded system matrix $\tilde{A}=V A U+M$ is then (13) shown at the bottom of the page. A similar structure can be written for the expanded control matrix $\tilde{B}=V B Q+N$.

From (12), we may identify the following particular cases: a) when $M_{12}=0, M_{23}+M_{33}=0$ and $M_{42}=0$; b) when $M_{21}=0, M_{22}+$ $M_{23}=0, M_{24}=0, N_{21}=0, N_{22}+N_{23}=0$ and $N_{24}=0$; and

$$
\tilde{A}=\left(\begin{array}{cccc}
A_{11} & \frac{1}{2} A_{12}+M_{12} & \frac{1}{2} A_{12}-M_{12} & A_{13} \\
A_{21}+M_{21} & \frac{1}{2} A_{22}+M_{22} & \frac{1}{2} A_{22}+M_{23} & A_{23}+M_{24} \\
A_{21}-M_{21} & \frac{1}{2} A_{22}-\left(M_{22}+M_{23}+M_{33}\right) & \frac{1}{2} A_{22}+M_{33} & A_{23}-M_{24} \\
A_{31} & \frac{1}{2} A_{32}+M_{42} & \frac{1}{2} A_{32}-M_{42} & A_{33}
\end{array}\right)
$$


c) otherwise. Cases a) and b) include the expansions corresponding to aggregations and restrictions, respectively [10], which are well known in the literature.

Choosing $M_{22}+M_{33}=0$, conditions (12) hold for all $i>2$. For $i=2$, they are

$$
\begin{aligned}
& \left(\begin{array}{c}
M_{12} \\
M_{23}+M_{33} \\
M_{42}
\end{array}\right)\left(\begin{array}{lll}
M_{21} & M_{22}+M_{23} & M_{24}
\end{array}\right)=0 \\
& \left(\begin{array}{c}
M_{12} \\
M_{23}+M_{33} \\
M_{42}
\end{array}\right)\left(\begin{array}{lll}
N_{21} & N_{22}+N_{23} & N_{24}
\end{array}\right)=0
\end{aligned}
$$

Choosing $M_{23}+M_{33}=0$ or $M_{22}+M_{23}=0$, two subcases of case c) are obtained:

Subcase $c_{1}$ ): $M_{23}=-M_{33}$. Then, relations (14) are

$$
\begin{gathered}
\left(\begin{array}{c}
M_{12} \\
0 \\
M_{42}
\end{array}\right)\left(\begin{array}{lll}
M_{21} & M_{22} & M_{24}
\end{array}\right)=0 \\
\left(\begin{array}{c}
M_{12} \\
0 \\
M_{42}
\end{array}\right)\left(\begin{array}{lll}
N_{21} & N_{22}+N_{23} & N_{24}
\end{array}\right)=0 .
\end{gathered}
$$

Subcase $\mathrm{c}_{2}$ ): $M_{23}=-M_{22}$. Then, relations (14) are

$$
\begin{gathered}
\left(\begin{array}{l}
M_{12} \\
M_{22} \\
M_{42}
\end{array}\right)\left(\begin{array}{lll}
M_{21} & 0 & M_{24}
\end{array}\right)=0 \\
\left(\begin{array}{l}
M_{12} \\
M_{22} \\
M_{42}
\end{array}\right)\left(\begin{array}{lll}
N_{21} & N_{22}+N_{23} & N_{24}
\end{array}\right)=0
\end{gathered}
$$

The above cases give possible structures to choose $M$ and $N$. A similar track should be followed to obtain structures for matrices $M_{Q^{*}}, N_{R^{*}}$, and $F$. Details are in [10].

Finally, the designer can select by optimization specific values of free elements of the block complementary matrices in (15) or (16) according to given design requirements. In the next section this procedure is illustrated in the context of design of overlapping decentralized controllers.

\section{EXAMPLE}

\section{A. Objective}

Consider the problem (1) for system (6) with the specific matrices

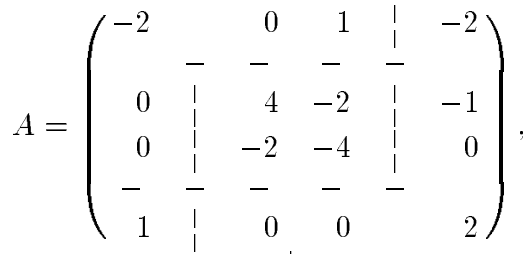

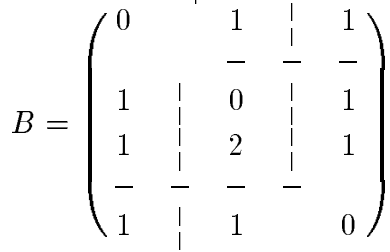

and $Q^{*}=\operatorname{diag}(1,1,1,1), R^{*}=\operatorname{diag}(1,1,1)$. The overlapping decomposition is determined by dashed lines. The pair $(A, B)$ is controllable.

The objective is to illustrate the potential advantages that offer the characterization of the complementary matrices presented above for an overlapping decentralized state LQ optimal control design.

We consider the following scheme: (1) the pair $(\mathbf{S}, J)$ is expanded to $(\tilde{\mathbf{S}}, \tilde{J})$; (2) a decentralized optimal control is designed for the decoupled expanded system in the form $\tilde{u}=-\tilde{K} \tilde{x}$, where $\tilde{K}$ is a block diagonal matrix; and (3) this control is contracted to be implemented in the original system $\mathbf{S}$ as $u=-K x$, where $K=Q \tilde{K} V$ according to Theorem 5. The evaluation of this controller is made by means of the suboptimality concept [7], [11], which is determined by the value $J^{\oplus}$ of the cost function in (1) for this controller. In order to eliminate the dependence of $J^{\oplus}$ on the initial state $x_{0}$, it is possible to assume $x_{0}$ as a random variable uniformly distributed over the $n$ dimensional unit sphere. Then, the expected value of the performance criterion is $\hat{J}^{\oplus}=\operatorname{Tr}\{H\}$, where $\operatorname{Tr}\{\cdot\}$ denotes the trace operator [9]. Matrix $H$ satisfies the Lyapunov equation $(A-B K)^{t} H+H(A-B K)+$ $K^{t} R^{*} K+Q^{*}=0$.

Let us consider the complementary matrices $N=0, M_{Q^{*}}=$ $0, N_{R^{*}}=0$, and $F=0$, which are particular simple cases that verify Theorems 4 and 6 . We select $M$ corresponding to the four cases described in the previous section and compute $\hat{J}^{\oplus}$. Since a goal of a decentralized control is to get responses as close as possible to the centralized control design, we consider the best case as the one leading to the minimum value of $\hat{J}^{\oplus}$.

\section{B. Results}

Case a) Overlapping Decomposition by Using an Aggregation: Here we choose the most typical matrix $M$ used in the literature [7], [11], that is

$$
\begin{aligned}
M & =\left(\begin{array}{ccccc}
0 & 0 & 0 & 0 \\
A_{21} & \frac{1}{2} A_{22} & -\frac{1}{2} A_{22} & -A_{23} \\
-A_{21} & -\frac{1}{2} A_{22} & \frac{1}{2} A_{22} & A_{23} \\
0 & 0 & 0 & 0
\end{array}\right) \\
& =\left(\begin{array}{rrrrrr}
0 & 0 & 0 & 0 & 0 & 0 \\
0 & 2 & -1 & -2 & 1 & 1 \\
0 & -1 & -2 & 1 & 2 & 0 \\
0 & -2 & 1 & 2 & -1 & -1 \\
0 & 1 & 2 & -1 & -2 & 0 \\
0 & 0 & 0 & 0 & 0 & 0
\end{array}\right)
\end{aligned}
$$

and the corresponding expanded matrix

$$
\begin{aligned}
\tilde{A} & =\left(\begin{array}{ccccc}
A_{11} & \frac{1}{2} A_{12} & \frac{1}{2} A_{12} & A_{13} \\
2 A_{21} & A_{22} & 0 & 0 \\
0 & 0 & A_{22} & 2 A_{23} \\
A_{31} & \frac{1}{2} A_{32} & \frac{1}{2} A_{32} & A_{33}
\end{array}\right) \\
& =\left(\begin{array}{rrrrrr}
-2 & 0 & 0.5 & 0 & 0.5 & -2 \\
0 & 4 & -2 & 0 & 0 & 0 \\
0 & -2 & -4 & 0 & 0 & 0 \\
0 & 0 & 0 & 4 & -2 & -2 \\
0 & 0 & 0 & -2 & -4 & 0 \\
1 & 0 & 0 & 0 & 0 & 2
\end{array}\right) .
\end{aligned}
$$

The computed suboptimal performance index is in this case $\hat{J}^{\oplus}=13.63$. 
Case b) Overlapping Decomposition by Using a Restriction: The typical choice of $M$ is in this case

$$
\begin{aligned}
M & =\left(\begin{array}{rrrrr}
0 & \frac{1}{2} A_{12} & -\frac{1}{2} A_{12} & 0 \\
0 & \frac{1}{2} A_{22} & -\frac{1}{2} A_{22} & 0 \\
0 & -\frac{1}{2} A_{22} & \frac{1}{2} A_{22} & 0 \\
0 & -\frac{1}{2} A_{32} & \frac{1}{2} A_{32} & 0
\end{array}\right) \\
& =\left(\begin{array}{rrrrrr}
0 & 0 & 0.5 & 0 & -0.5 & 0 \\
0 & 2 & -1 & -2 & 1 & 0 \\
0 & -1 & -2 & 1 & 2 & 0 \\
0 & -2 & 1 & 2 & -1 & 0 \\
0 & 1 & 2 & -1 & -2 & 0 \\
0 & 0 & 0 & 0 & 0 & 0
\end{array}\right)
\end{aligned}
$$

and the corresponding expanded matrix

$$
\begin{aligned}
\tilde{A} & =\left(\begin{array}{rccc}
A_{11} & A_{12} & 0 & A_{13} \\
A_{21} & A_{22} & 0 & A_{23} \\
A_{21} & 0 & A_{22} & A_{23} \\
A_{31} & 0 & A_{32} & A_{33}
\end{array}\right) \\
& =\left(\begin{array}{rrrrrr}
-2 & 0 & 1 & 0 & 0 & -2 \\
0 & 4 & -2 & 0 & 0 & -1 \\
0 & -2 & -4 & 0 & 0 & 0 \\
0 & 0 & 0 & 4 & -2 & -1 \\
0 & 0 & 0 & -2 & -4 & 0 \\
1 & 0 & 0 & 0 & 0 & 2
\end{array}\right) .
\end{aligned}
$$

The suboptimal performance index is $\hat{J}^{\oplus}=15.44$.

Subcase $c_{1}$ ) Overlapping Decomposition by Using the Proposed Method: With the purpose of having as many zeros as possible in the off-diagonal blocks of the expanded matrix (13), we can select the complementary submatrices of $M$ as $M_{12}=(1 / 2) A_{12}=$ (0 $(1 / 2)), M_{21}=A_{21}=\left(\begin{array}{l}0 \\ 0\end{array}\right), M_{24}=-A_{23}=$ $\left(\begin{array}{l}1 \\ 0\end{array}\right), M_{42}=-(1 / 2) A_{32}=\left(\begin{array}{ll}0 & 0\end{array}\right)$.

If $M_{12} M_{22}=0$, the relations (15) hold. Denote $M_{22}=\left(\begin{array}{cc}m_{22} & m_{23} \\ 0 & 0\end{array}\right)$. Now, we have the degree of freedom to select the values of $m_{22}$ and $m_{23}$. We use an algorithm of steepest descent gradient method to minimize $\hat{J}^{\oplus}$ with respect to $m_{22}$ and $m_{23}$, which solves the Lyapunov equation using MATLAB. Thus, we obtain the submatrix $M_{22}=\left(\begin{array}{cc}-0.22 & -0.82 \\ 0 & 0\end{array}\right)$. The complete matrices $M$ and $\tilde{A}$ given by (11) and (13), respectively, are the following:

$$
\begin{gathered}
M=\left(\begin{array}{cccccr}
0 & 0 & 0.50 & 0 & -0.50 & 0 \\
0 & -0.22 & -0.82 & -0.22 & -0.82 & 1 \\
0 & 0 & 0 & 0 & 0 & 0 \\
0 & 0.22 & 0.82 & 0.22 & 0.82 & -1 \\
0 & 0 & 0 & 0 & 0 & 0 \\
0 & 0 & 0 & 0 & 0 & 0
\end{array}\right), \\
\tilde{A}=\left(\begin{array}{cccccr}
-2 & 0 & 1 & 0 & 0 & -2 \\
0 & 1.78 & -1.82 & 1.78 & -1.82 & 0 \\
0 & -1 & -2 & -1 & -2 & 0 \\
0 & 2.22 & -0.18 & 2.22 & -0.18 & -2 \\
0 & -1 & -2 & -1 & -2 & 0 \\
1 & 0 & 0 & 0 & 0 & 2
\end{array}\right) .
\end{gathered}
$$

The computed suboptimal performance index is $\hat{J}^{\oplus}=$ 11.02 .
Subcase $c_{2}$ ) Overlapping Decomposition by Using the Proposed Method: The complementary submatrices $M_{12}, M_{21}, M_{24}$, and $M_{42}$ are the same as in subcase $\left.\mathrm{c}_{1}\right)$. The submatrix $M_{22}$ can be selected as $M_{22}=\left(\begin{array}{ll}0 & m_{23} \\ 0 & m_{33}\end{array}\right)$ to satisfy relations (16). In this situation, the minimization algorithm gives the solution $M_{22}=\left(\begin{array}{cc}0 & -0.41 \\ 0 & 2.01\end{array}\right)$. Thus, $M$ and $\tilde{A}$ are

$$
\begin{aligned}
M & =\left(\begin{array}{rrrrrc}
0 & 0 & 0.50 & 0 & -0.50 & 0 \\
0 & 0 & -0.41 & 0 & 0.41 & 1 \\
0 & 0 & 2.01 & 0 & -2.01 & 0 \\
0 & 0 & -0.41 & 0 & 0.41 & -1 \\
0 & 0 & 2.01 & 0 & -2.01 & 0 \\
0 & 0 & 0 & 0 & 0 & 0
\end{array}\right) \\
\tilde{A} & =\left(\begin{array}{rrrrrr}
-2 & 0 & 1 & 0 & 0 & -2 \\
0 & 2 & -1.41 & 2 & -0.59 & 0 \\
0 & -1 & 0.01 & -1 & -4.01 & 0 \\
0 & 2 & -1.41 & 2 & -0.59 & -2 \\
0 & -1 & 0.01 & -1 & -4.01 & 0 \\
1 & 0 & 0 & 0 & 0 & 2
\end{array}\right) .
\end{aligned}
$$

The computed suboptimal performance index is $\hat{J}^{\oplus}=$ 10.48 .

\section{Remark}

The centralized optimal control for the initial system $\mathbf{S}$ in (1) results in $\hat{J}^{\circ}=9.88$. We can observe that the control obtained through the expansions defined by the proposed strategy approaches closer to this value than those obtained via the usual aggregations or restrictions. These results illustrate the degree of freedom introduced by this approach to select the complementary matrices in minimizing the suboptimality in overlapping decentralized control design. This minimization has been performed here with respect to two elements of the matrix $M$ only for illustrative purposes. The same objective can be considered with respect to more elements in $M$ and other complementary matrices.

\section{CONCLUSION}

The introduction of appropriate changes of basis in the expansioncontraction process has been the starting point of this paper to give a new general block structure characterization of the complementary matrices involved in the Inclusion Principle for state LQ optimal control when considering LTI systems. The identified block structure gives a framework strategy for choosing the complementary matrices in a more flexible manner that if one directly uses the fundamental theorems of the Inclusion Principle. This flexibility has been illustrated in this paper in the context of overlapping decentralized LQ optimal control through the selection of one of the complementary matrices with the objective of minimizing the suboptimality degree. In this context, a more involved optimization problem with respect to more complementary matrices can be pursued in future works. Moreover, the degree of freedom offered by the identified block structure can be exploited in expansion-contraction schemes to select complementary matrices with respect to other design objectives.

\section{REFERENCES}

[1] L. Bakule and J. Lunze, "Decentralized design of feedback control for large-scale systems," Kybernetika, vol. 3-6, pp. 1-100, 1988.

[2] L. Bakule and J. Rodellar, "Decentralized control and overlapping decomposition of mechanical systems. Part 1: System decomposition. Part 2: Decentralized stabilization," Int. J. Contr., vol. 61, pp. 559-587, 1995. 
[3] L. Bakule, J. Rodellar, and J. M. Rossell, "Structure of expansion-contraction matrices in the inclusion principle for dynamic systems," SIAM J. Matrix Analysis Applicat., vol. 21, no. 4, pp. 1136-1155, 2000.

[4] M. Ikeda and D. D. Siljak, "Overlapping decompositions, expansions and contractions of dynamic systems," Large Scale Syst., vol. 1, pp. $29-38,1980$.

[5] — - "Generalized decompositions of dynamic systems and vector Lyapunov funtions," IEEE Trans. Automat. Contr., vol. AC-26, pp. $1118-1125,1981$.

[6] - "Overlapping decentralized control with input, state and output inclusion," Control-Theory and Advanced Technol., vol. 2, pp. 155-172, 1986.
[7] M. Ikeda, D. D. Šiljak, and D. E. White, "Decentralized control with overlapping informations sets," JOTA, vol. 34, no. 2, pp. 279-309, 1981.

[8] —, "An inclusion principle for dynamic systems," IEEE Trans. Automat. Contr., vol. AC-29, pp. 244-249, 1984

[9] W. S. Levine and M. Athans, "On the determination of the optimal constant output feedback gains for linear multivariable systems," IEEE Trans. Automat. Contr., vol. AC-15, pp. 44-48, 1970.

[10] J. M. Rossell, "Contribution to decentralized control of large-scale systems via overlapping models," Ph.D. dissertation, Tech. Univ. Catalunya, Barcelona, Spain, 1998. (in Spanish).

[11] D. D. Siljak, Decentralized Control of Complex Systems. New York: Academic, 1991. 